\title{
Asymptotic Behaviour of a Thermosyphon model with binary fluids
}

\author{
Ángela Jiménez-Casas
}

Grupo Dinámica No lineal, Universidad Pontificia Comillas. Madrid (Spain), ajimenez@ comillas.edu

\begin{abstract}
The aim of this work is the generalization for a thermosyphon model where on be considered a binary fluid, of the theoretical results about the asymptotic behaviour of the thermosyphon model for a fluid with one-component from ([10]).
\end{abstract}

\section{INTRODUCTION}

The concern of this study is to analyze the dynamics of a fluid transporting an inner solute along an arbitrary close geometry and subject to gravity and convection forces (thermosyphon). In the present work will be considered the thermosyphon model with a binary fluid - fluid with a solute substance -, and we study the evolution of the velocity $v$, the temperature of the fluid, $T$, and the solute concentration, $S$.

In this case the Soret's effect is considered, which consists in a solute flux generation due to a temperature gradient in the fluid (see [1],[7],[13]). The physical reason for this effect seems to rest on the system auto-organization in a manner to get the most efficient heat transport (minimum entropy production). In general, the heaviest specie for the one with the strongest interaction is accumulated in the coldest system region. The Soret's effect is mathematically represented as a first approximation by a coefficient ratio product and the temperature gradient, this coefficient is denoted by Soret's coefficient.

The evolution of the above quantities is given by the following coupled ODE/PDE system (see [6], [4], [8], [3], [12] for further details) with periodic boundary conditions for $T, S$

$$
\left\{\begin{array}{c}
\varepsilon \frac{d v}{d t}+G(v) v=\oint[T-S] f(x) d x, v(0)=v_{0} \\
\frac{\partial T}{\partial t}+v \frac{\partial T}{\partial x}=H(v)\left(T_{a}-T\right), T(0, x)=T_{0}(x) \\
\frac{\partial S}{\partial t}+v \frac{\partial S}{\partial x}=c \frac{\partial^{2} S}{\partial x^{2}}-b \frac{\partial^{2} T}{\partial x^{2}}, S(0, x)=S_{0}(x)
\end{array}\right.
$$

We assume that the section of the loop is constant and small compared with the dimension of the physical circuit, so that the arc length co-ordinate along the loop $x$ gives the position in the circuit. The velocity is assumed to be independent of the position in the circuit.

The function $f$ describes the geometry of the loop and the distribution of gravitational forces, $\oint=\int_{0}^{1} d x$ denotes the path integration along the closed path of the circuit. We assume that $G(v)$, which specifies the frictional law at the inner wall of the loop, and $H$, associated to the heat flux across the loop wall, are positive and bounded away from zero, with $G(v) \geq G_{0}>0, H(v) \geq H_{0}>0$.

We consider $G, f$ and $H$ are given functions together with the constants, $b, c>0$, and the ambient temperature, $T_{a}(x)$. The constant $b$ is proportional to the Soret's coefficient ([1]). [4]).

We note that $\oint f=0$ and all functions depending on $x$ are 1-periodic respect to $x$ and have zero average (see [6],

The result about the well-posedness and asymptotic behaviour for the system (1) it can be found in ([6], [4]). In this previous works the existence and uniqueness of solution is proved for every time with initial data in suitable framework (see also [5]). In this previous works have been studied the asymptotic behaviour of the system (1) when time goes to infinity.

In this sense has been proved the existence of a inertial manifold associated to the functions $f$ (loop-geometry) and $T_{a}$ (ambient temperature), using the the abstract operators theory ([2], [11]). 
We assume that $G^{*}(r)=r G(r)$ is locally Lipschitz, $H \in C^{1}, T_{a} \in \dot{H}_{p e r}^{2}$ and $f \in \dot{L}_{p e r}^{2}$ are given by following Fourier expansions

$$
T_{a}(x)=\sum_{k \in Z^{*}} b_{k} e^{2 \pi k i x} ; \quad f(x)=\sum_{k \in Z^{*}} c_{k} e^{2 \pi k i x} ; \text { where } Z^{*}=Z-\{0\},
$$

while $T_{0} \in \dot{H}_{p e r}^{2}$ and $S_{0} \in \dot{L}_{p e r}^{2}$ are given by

$$
T_{0}(x)=\sum_{k \in Z^{*}} a_{k 0} e^{2 \pi k i x}, S_{0}(x)=\sum_{k \in Z^{*}} d_{k 0} e^{2 \pi k i x} .
$$

Finally assume that $T(t, x) \in \dot{H}_{p e r}^{2}$ and $S(t, x) \in \dot{L}_{p e r}^{2}$ are given by

$$
T(t, x)=\sum_{k \in Z^{*}} a_{k}(t) e^{2 \pi k i x} \text { and } S(t, x)=\sum_{k \in Z^{*}} d_{k}(t) e^{2 \pi k i x} \quad Z^{*}=Z-\{0\}
$$

We note that $\bar{a}_{k}=-a_{k}\left(\bar{d}_{k}=-d_{k}\right)$ since all functions consider are real and also $a_{0}=d_{0}=0$ since they have zero average.

Now we observe the dynamics of each Fourier mode and from (1), we get the following system for the new unknowns, $v$ and the coefficients $a_{k}(t)$ and $d_{k}(t)$.

$$
\left\{\begin{array}{c}
\varepsilon \frac{d v}{d t}+G(v) v=\sum_{k \in Z^{*}}\left(a_{k}(t)-d_{k}(t)\right) c_{-k} \\
\dot{a}_{k}(t)+[2 \pi k i v(t)+H(v(t))] a_{k}(t)=H(v(t)) b_{k} \\
\dot{d}_{k}(t)+\left[2 \pi k i v(t)+4 c \pi^{2} k^{2}\right] d_{k}(t)=-4 b \pi^{2} k^{2} a_{k}(t)
\end{array}\right.
$$

We consider the functions $T_{a}$ and $f$ are given by following Fourier expansions

$$
\begin{gathered}
T_{a}(x)=\sum_{k \in K} b_{k} e^{2 \pi k i x} ; \quad f(x)=\sum_{k \in J} c_{k} e^{2 \pi k i x} ; \text { where } J=\left\{k \in Z^{*} / c_{k} \neq 0\right\}, K=\left\{k \in Z^{*} / b_{k} \neq 0\right\} \text { with } Z^{*}=Z-\{0\}, \\
\text { and } \oint(T-S) f=\sum_{k \in(K \cap J)}\left(a_{k}(t)-d_{k}(t)\right) c_{-k} .
\end{gathered}
$$

We prove working with the equations (4) the velocity of the fluid is independent of the coefficients for temperature $a_{k}(t)$ and the salinity $d_{k}(t)$ for every $k \in Z^{*}-(K \cap J)$. That is, the relevant coefficients for the evolution of the velocity are only $a_{k}(t)$ and $d_{k}(t)$ with $k$ belonging to the set $K \cap J$. This important result about the asymptotic behaviour has been proved in ([6], [4]) using the inertial manifold theory ([2], [11]).

\section{ASYMPTOTIC BEHAVIOUR FOR SMALL OF PARAMETER $\varepsilon$}

In this section the fluid velocity behaviour is studied under the assumption of small values of the parameter $\varepsilon$, which is related to the ratio between the effects of the fluid acceleration due to viscous forces and the acceleration due to the effect of temperature in the fluid density (origin of the Soret effect). Considering both effects of the same order to enable one to consider the Soret effect (relevant effect in the thermosyphon problem), there results a direct association between the parameter $\varepsilon$ and the fluid inertia acceleration; therefore small values of the parameter means small fluid acceleration, which involves a behaviour close to the stationary regime (result to be proved theoretically in [6]).

We shall consider the case where the functions $H$ and $G$ depend on $\varepsilon$, i.e. $G=H=H_{\varepsilon}(v)=\frac{H_{0}(v)}{\varepsilon}$, and we shall study the system behaviour for small values of $\varepsilon$.

The aim is to show, Proposition 2, that for sufficient small values of $\varepsilon$ every solution behaves as a stationary solution with null velocity.

Now, the reduced system behaviour is studied, equivalent to whole system in the asymptotic behaviour in the large time, as it was shown above.

Lemma If we assume that $\int_{0}^{\infty}|v(s)| d s<\infty$, then for every $\delta>0$ there exists $t_{r}$ such that

$$
\int_{0}^{t} H(r) e^{-\int_{r}^{t} H}\left(e^{-\int_{r}^{t} 2 \pi i k v}-1\right) d r \leq \eta \quad \forall \eta \text { with } t \geq t_{r}
$$


Proof: If $\int_{0}^{\infty}|v(s)| d s<\infty$, it means that $\forall \delta, \exists t_{r}(\delta)>0 /\left|\int_{r}^{t} v\right| \leq \delta$ if $t_{r} \leq r \leq t<\infty$. Thus, $\forall \delta$ there exists $t>0$ such that $\left|\int_{r}^{t} v\right| \leq \delta$ and then there exists $t_{r} /\left|e^{-\int_{r}^{t} 2 \pi i k v}-1\right| \leq \eta \forall t \geq t_{r}$ and therefore $\int_{0}^{t} H(r) e^{-\int_{r}^{t} H}\left(e^{-\int_{r}^{t} 2 \pi i k v}-1\right) d r \leq$ $\eta\left(1-e^{-\int_{r}^{t} H}\right) \leq \eta, \forall \eta$ with $t \geq t_{r}$, taking into account that $\eta \rightarrow 0$ for $t \rightarrow \infty$ and $H$ is strictly bounded away from zero, we get (6).

Proposition 1 i) If $I=\sum_{k \in K \cap J} b_{k} c_{-k}=\oint T_{a} f=0$, and $\int_{0}^{\infty}|v(s)| d s<\infty$, then the system goes to the rest stationary solution, that is to say:

$$
\left(v(t), a_{k}(t), d_{k}(t)\right) \rightarrow\left(0, b_{k}, \frac{b}{c} b_{k}\right), \text { i.e. } \quad(v(t), T(t), S(t)) \rightarrow\left(0, T_{a}, \frac{b}{c} T_{a}\right)
$$

ii) If $I=\sum_{k \in K \cap J} b_{k} c_{-k} \neq 0$ then $\int_{0}^{\infty}|v(s)| d s=\infty$, and $v(t)$ does not converge to zero.

Proof:(see [6])

However, this last result can be refined showing the coefficients tendency to their equilibrium values as a function of the parameter under study, $\varepsilon$, as following:

Proposition 2 If $I=\sum_{k \in K \cap J} b_{k} c_{-k}=0$, and $\int_{0}^{\infty}|v(s)| d s<\infty$, then

$$
\begin{gathered}
\limsup _{t \rightarrow \infty}\left|a_{k}(t)-b_{k}\right| \leq \varepsilon 2 \pi|k|\left|b_{k}\right| \limsup _{t \rightarrow \infty} \frac{|v(t)|}{H_{0}(v(t))} \\
\limsup _{t \rightarrow \infty}\left|d_{k}(t)-\frac{b}{c} b_{k}\right| \leq \varepsilon\left(4 c \pi^{2} k^{2}\right) 2 \pi\left|k \| b_{k}\right| \limsup _{t \rightarrow \infty} \frac{|v(t)|}{H_{0}(v(t))}
\end{gathered}
$$

and

$$
\begin{gathered}
\limsup _{t \rightarrow \infty}|v(t)| \leq \varepsilon 2 \pi \sum_{K \cap J}|k|\left|b_{k} \| c_{-k}\right| \limsup _{t \rightarrow \infty} \frac{1}{G(v(t))}+ \\
+\varepsilon 8 c \pi^{3} \sum_{K \cap J}|k|^{3}\left|b_{k}\right|\left|c_{-k}\right| \limsup _{t \rightarrow \infty} \frac{1}{G(v(t))}
\end{gathered}
$$

Proof: To study $a_{k}(t)$ we defined $\widehat{a_{k}}=b_{k} \int_{0}^{t} H(r) e^{-\int_{r}^{t} H_{\varepsilon}} d r=b_{k}\left(1-e^{-\int_{0}^{t} H_{\varepsilon}}\right)$ that converges to $b_{k}$ for $t \rightarrow \infty$ $\left(\lim _{t \rightarrow \infty} \widehat{a_{k}}=b_{k}\right)$, and defining $\tau_{k}(t)=a_{k}(t)-\widehat{a_{k}}, \tau_{k}$ satisfies,

$$
\tau_{k}(t)=a_{k}(0) e^{-\int_{0}^{t}\left(2 \pi k v i+H_{\varepsilon}\right)}+b_{k} \int_{0}^{t} e^{-\int_{r}^{t} H_{\varepsilon}}\left(e^{-\int_{r}^{t} 2 \pi k v i}-1\right) H_{\varepsilon}(r) d r
$$

taking modulus and applying the triangular inequality, we get

$$
\left|\tau_{k}(t)\right| \leq\left|a_{k}(0)\right| e^{-\int_{0}^{t} H_{\varepsilon}}+\left|b_{k}\right| \frac{\int_{0}^{t} H_{\varepsilon}(r) e^{\int_{0}^{r} H_{\varepsilon}}\left|e^{-\int_{r}^{t} 2 \pi k i v}-1\right| d r}{e^{\int_{0}^{t} H_{\varepsilon}}}
$$

since $H$ is strictly bounded away from zero together with Lemma 1, we get,

$$
\left|\tau_{k}(t)\right| \leq\left|a_{k}(0)\right| e^{-\left(H_{\varepsilon}\right)_{0} t}+2 \pi|k|\left|b_{k}\right| \frac{\int_{0}^{t} H_{\varepsilon}(r) e^{\int_{0}^{r} H_{\varepsilon}} \int_{r}^{t}|v| d r}{e^{\int_{0}^{t} H_{\varepsilon}}}
$$

taking limits when $t \rightarrow \infty$,

$$
\limsup _{t \rightarrow \infty}\left|\tau_{k}(t)\right| \leq 2 \pi|k|\left|b_{k}\right| \limsup \sin _{t \rightarrow \infty} \frac{\int_{0}^{t} H_{\varepsilon}(r) e^{\int_{0}^{r} H_{\varepsilon}} \int_{r}^{t}|v| d r}{e^{\int_{0}^{t} H_{\varepsilon}}}
$$

and applying the L'Hopital Lemma, it is obtained

$$
\limsup _{t \rightarrow \infty}\left|\tau_{k}(t)\right| \leq \varepsilon 2 \pi|k|\left|b_{k}\right| \limsup \sin _{t \rightarrow \infty} \frac{|v(t)|}{H_{0}(v(t))}\left(1-e^{-\int_{0}^{t} H_{\varepsilon}}\right)
$$


and we obtain (7). For $d_{k}(t)$ we define $\widehat{d_{k}}=\left(1-e^{-4 c \pi^{2} k^{2}\left(t-t_{0}\right)}\right) \frac{b}{c} b_{k}$ that converges to $\frac{b}{c} b_{k}$ for $t \rightarrow \infty\left(\lim _{t \rightarrow \infty} \widehat{d_{k}}=\frac{b}{c} b_{k}\right)$, and defining $\sigma_{k}(t)=d_{k}(t)-\widehat{d}_{k}$, thus $\sigma_{k}$ satisfies,

$$
\sigma_{k}(t)=d_{k}(0) e^{-\int_{0}^{t}\left(2 \pi k v i+4 c \pi^{2} k^{2}\right)}+\int_{0}^{t}\left(-4 c \pi^{2} k^{2}\right) e^{-4 c \pi^{2} k^{2}(t-r)} \frac{b}{c}\left(a_{k}(r) e^{-\int_{r}^{t} 2 \pi k v i}-b_{k}\right) d r .
$$

Now, from Lemma 1, and considering (7), we get (8). Next, reading the equation for $v$ as

$$
\varepsilon \frac{d v}{d t}+G(v) v=\left(I_{1}(t)-I\right)+\left(I_{2}(t)-\frac{b}{c} I\right)+\left(1+\frac{b}{c}\right) I
$$

where $I_{1}(t)=\sum_{k \in K \cap J} a_{k}(t) c_{-k}$ and $I_{2}(t)=\sum_{k \in K \cap J} d_{k}(t) c_{-k}$, we get

$$
\begin{gathered}
v(t)=v_{0} e^{-\int_{0}^{t} G_{\varepsilon}(v)}+\frac{1}{\varepsilon} \int_{0}^{t}\left(I_{1}(t)-I\right) e^{-\int_{r}^{t} G_{\varepsilon}(v)} d r+\frac{1}{\varepsilon} \int_{0}^{t} I e^{-\int_{r}^{t} G_{\varepsilon}(v)} d r+ \\
+\frac{1}{\varepsilon} \int_{0}^{t}\left(I_{2}(t)-\frac{b}{c} I\right) e^{-\int_{r}^{t} G_{\varepsilon}(v)} d r+\frac{b}{c \varepsilon} \int_{0}^{t} I e^{-\int_{r}^{t} G_{\varepsilon}(v)} d r .
\end{gathered}
$$

Finally, we have that for every $\delta>0$ there exists $t_{0}$ such that $\left|I_{1}(t)-I\right| \leq \varepsilon M+\delta$ and $\left|I_{2}(t)-\frac{b}{c} I\right| \leq \varepsilon N+\delta$, where $M=2 \pi \sum_{K \cap J}|k|\left|b_{k}\right|\left|c_{-k}\right|$ and $N=8 c \pi^{2} \sum_{K \cap J}|k|^{3}\left|b_{k}\right|\left|c_{-k}\right|$ and using again L'Hopital's Lemma for the function $F(t)=\int_{0}^{t} \frac{1}{\varepsilon} e^{-\int_{r}^{t} G_{\varepsilon}} d r$, and from (7) together with (8), we get (for $I=0$ ) the estimates on the limit of $v|(t)|$ and we conclude. $\square$

\section{ACKNOWLEDGMENTS}

This research was partially supported by grants MTM2012-31298,MTM2016-75465-P and Project FIS2013-47949-C2-2 from Ministerio de Economia y Competitividad, Spain; by GR58/08 Grupo 920894 BSCH-UCM from Grupo de Investigacion CADEDIF, Grupo de Dinamica No Lineal (U.P. Comillas)Spain and by the Project FIS2016-78883-C2-2-P (AEI/FEDER, UE).

\section{REFERENCES}

[1] J.E. Hart, "A Model of Flow in a Closed-Loop Thermosyphon including the Soret Effect", J. of Heat Transfer, 107, 840-849, (1985).

[2] D. Henry, "Geometric Theory of Semilinear Parabolic Equations", Lectures Notes in Mathematics 840, Springer-Verlag, (1981).

[3] M.A. Herrero, J.J-L. Velazquez, "Stability analysis of a closed thermosyphon”, European J. Appl. Math., 1, $1-24,(1990)$.

[4] A. Jiménez-Casas, A. Rodríguez-Bernal, "Finite-dimensional asymptotic behavior in a thermosyphon including the Soret effect", Math. Meth. in the Appl. Sci., 22, 117-137 (1999).

[5] A. Jiménez-Casas"A coupled ODE/PDE system governing a thermosyphon model”, Nonlinear Analysis, 47, 687-692 (2001).

[6] A. Jiménez Casas, "Well posedness and asymptotic behaviour of a closed loop thermosyphon in Spectral Theory and Nonlinear Ana ", Spectral Theory and Nonlinear Analysis with Applications to Spatial Ecology, World Scientific Publishing, 59-74, ISBN 981-256-514-0 (2005).

[7] J.B. Keller, "Periodic oscillations in a model of thermal convection", J. Fluid Mech., 26, 3, 599-606, (1966).

[8] A. Liñan, "Analytical description of chaotic oscillations in a toroidal thermosyphon", in Fluid Physics, Lecture Notes of Summer Schools, (M.G. Velarde, C.I. Christov, Eds.) pp. 507-523, World Scientific, Singapore, (1994).

[9] A. Rodríguez-Bernal, E.S. Van Vleck, "Diffusion Induced Chaos in a Closed Loop Thermosyphon", SIAM J. Appl Math., vol. 58, 4, 1072-1093, (1998).

[10] A. Rodríguez-Bernal, E. S. Van Vleck, "Complex oscillations in a closed thermosyphon", in Int. J. Bif. Chaos, vol. 8, 1, 41-56 (1998).

[11] R. Temam, "Infinite Dimensional Dynamical Systems in Mechanics and Physics", Appl. Math. Sci. 68, Springer-Verlag, New York, (1988).

[12] J.J.L. Velázquez, "On the dynamics of a closed thermosyphon”, SIAM J.Appl. Math. 54, $n^{\circ}$ 6, 1561-1593, (1994).

[13] P. Welander, "On the oscillatory instability of a differentially heated fluid loop," J. Fluid Mech. 29, No 1, 17-30 (1967). 